\title{
Polypoidal choroidal vasculopathy-associated vitreous haemorrhage presenting as hyphema
}

\author{
Srikanta Kumar Padhy, Atul Kumar, Rebika Dhiman, Karen Sharma
}

Dr Rajendra Prasad Centre for Ophthalmic Sciences, All India Institute of Medical Sciences, New Delhi, Delhi, India

\section{Correspondence to} Dr Srikanta Kumar Padhy, srikantkumar.padhy19@gmail. com

Accepted 13 November 2018

\section{DESCRIPTION}

A 59-year-old Indian male patient presented to our clinic with sudden-onset, painless diminution of vision in the right eye for the last 1 week. Records from previous follow-up ( 2 months back) showed that his vision was 20/200 right eye (OD) and 20/80 left eye (OS). He had no known systemic medical conditions. He was diagnosed as a case of right eye pseudophakia with polypoidal choroidal vasculopathy (figure 1A), as evidenced by his previous fundus fluorescein angiography (FFA) (figure 1B), indocyanine green angiography (ICGA) (figure 1C) and swept source optical coherence tomography (SSOCT) (figure 2A,C). He has been on regular follow-up for the same condition for the past 4 years. During the course, he had received four intravitreal antivascular endothelial growth factor (anti-VEGF, aflibercept $2 \mathrm{mg}$ in $0.05 \mathrm{~mL}$ ) injections, with the last injection being administered 4 months back.

On ocular examination, his best-corrected visual acuity was hand movement close to face OD and 20/80 OS. Intraocular pressure was $16 \mathrm{~mm} \mathrm{Hg} \mathrm{OD}$ and $14 \mathrm{~mm} \mathrm{Hg}$ OS. On slit lamp examination, presence of hyphema (figure $3 \mathrm{~A}$ ) was noted in the right eye obscuring the details of the rest of the anterior segment. The anterior segment of the fellow eye was unremarkable. Ocular ultrasonography showed the presence of moderate amplitude spikes in the vitreous area suggestive of haemorrhage in the right eye (figure 3B). Dilated fundus evaluation of the fellow eye showed a sharply delineated area of geographic atrophy (hypopigmentation with apparent absence of retinal pigment epithelium (RPE) (figure 4A). Fundus autofluorescence imaging of the same showed sharply demarcated area of hypoautofluorescence (figure 4B) with window defect on FFA due to atrophy of the overlying RPE (figure 4C). ICGA excluded the presence

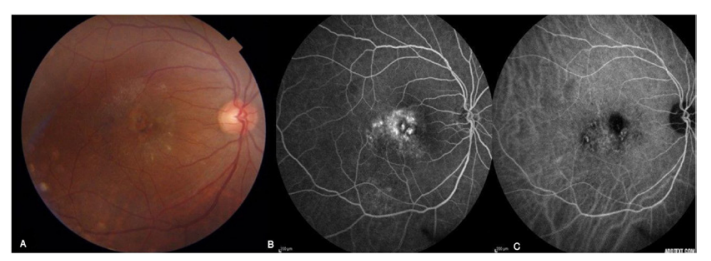

Figure 1 (A) Fundus photograph of the right eye showing polypoidal orange-red lesions in the macula. (B) Fundus fluorescein angiography of the same eye showing areas of hyperfluorescence in the macula due to leakage. (C) Indocyanine green angiography showing hypofluorescent polypoidal lesions with hyperfluorescent halo corresponding to the orange-red subretinal nodules.

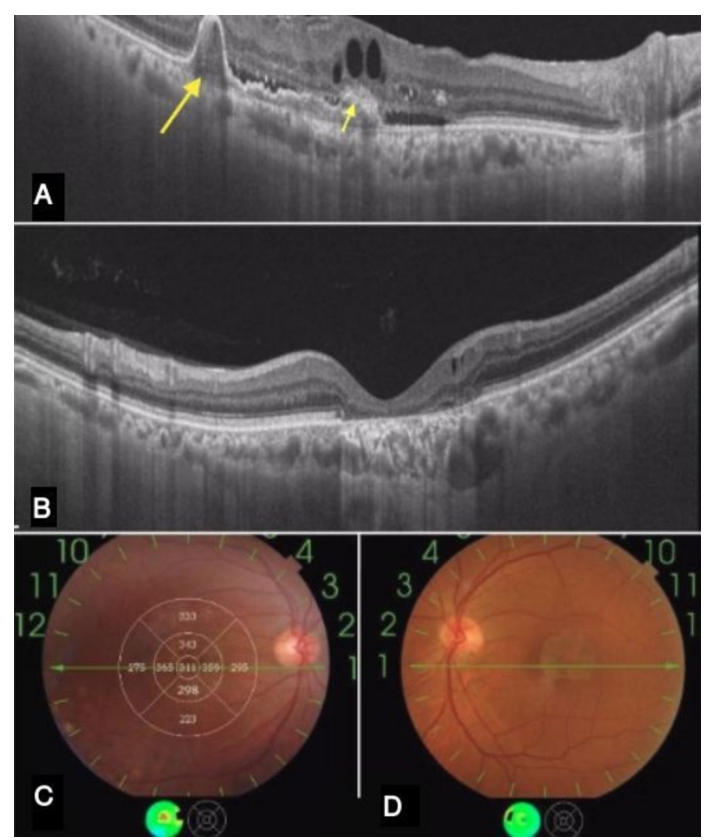

Figure $2(A, C)$ Swept source optical coherence tomography (SSOCT) imaging of the right eye showing thumb-shaped pigment epithelial detachment (long arrow) with double-layer sign (short arrow). (B,D) SSOCT of the left eye showing foveal atrophy.

of any secondary choroidal neovascular membrane in the left eye (figure 4D). SSOCT of the left eye (figure 2B,D) revealed thinning of the hyper-reflective external band due to RPE/Bruch's membrane complex attenuation. Thus, we made a diagnosis of right eye hyphema with vitreous haemorrhage (VH) secondary to polypoidal choroidal vasculopathy and left eye geographic atrophy.

In view of the normal intraocular pressure and the absence of endothelial staining, the patient was medically managed for hyphema with topical steroids (prednisolone acetate 1\%), mydriatic (homatropine 2\%) and antiglaucoma (timolol $0.5 \%$ ), and was given propped-up positioning. The patient is being followed up weekly. A surgical pars plana vitrectomy has been planned as the next step after the clearing of hyphema.

Polypoidal vasculopathy (PCV) is a disease of pachychoroid spectrum primarily involving the choroidal circulation. It is characterised by the aneurysmal bulge of the inner choroidal vessel endings, which are clinically visible as reddish-orange polyps. ${ }^{1}$ Spontaneous rupture of these venules and occasionally arteries is not uncommon, and may lead to massive breakthrough subretinal and/ or vitreous haemorrhage causing a sudden, painless 


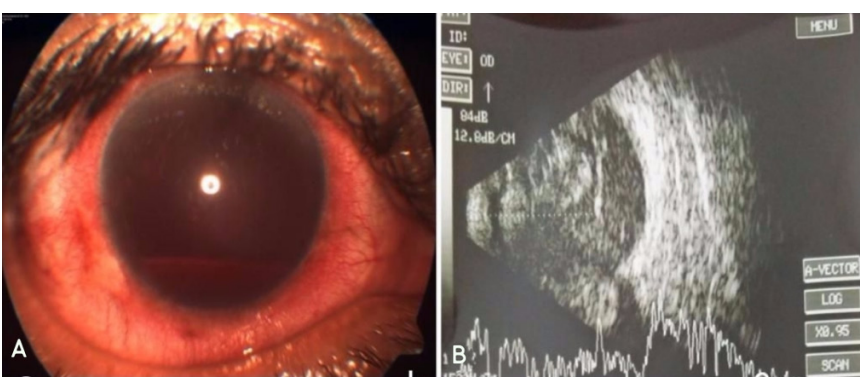

Figure 3 (A) Anterior segment photograph of the right eye showing hyphema on slit lamp examination. (B) Ocular ultrasonography of the right eye showing mild to moderate amplitude spikes suggestive of vitreous haemorrhage.

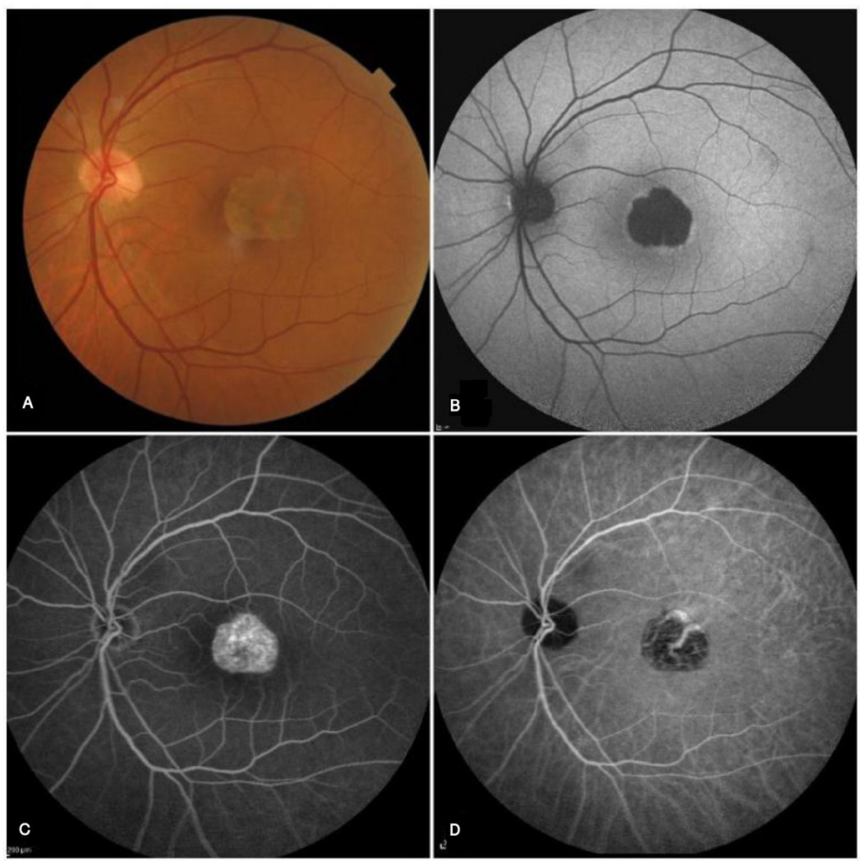

Figure 4 (A) Fundus photograph of the left eye showing a sharply delineated area of hypopigmentation in the macula suggestive of geographic atrophy. (B) Fundus autofluorescence image of the left eye showing sharply delineated area of hypoautofluorescence. (C) Fundus fluorescein angiography of the left eye showing a well-demarcated RPE window defect. (D) Indocyanine green angiography of the left eye showing absence of choroidal neovascular membrane. RPE, retinal pigment epithelium. vision loss. This can occur as the initial presentation of the disease or during the course of follow-up. PCV-related breakthrough $\mathrm{VH}$ can also develop following photodynamic therapy (PDT), intravitreal injection of anti-VEGF agents, pneumatic displacement of submacular haemorrhage, or combined therapy with PDT and anti-VEGF agents. ${ }^{2}$ The occurrence of breakthrough $\mathrm{VH}$, subretinal haemorrhage and suprachoroidal haemorrhage is known in PCV, but anterior chamber hyphema secondary to PCV-related VH has not previously been described. We presume that the amount of breakthrough $\mathrm{VH}$ in our case was extensive enough to pass through the weakened zonules in pseudophakic eye and appears in the anterior chamber. In this case, the breakthrough bleed was likely a spontaneous event, and not triggered by intravitreal injection that was administered 4 months before the bleed. VH due to intravitreal injection mostly occurs early (within 1-4 days of drug administration). ${ }^{2}$ There are variable reports on the visual outcome in patients undergoing vitrectomy for $\mathrm{VH}$ in PCV. ${ }^{12}$ Visual recovery after pars plana vitrectomy is mainly limited by macular involvement and the presence of subfoveal or juxtafoveal polyps. ${ }^{2}$

\section{Learning points}

- Polypoidal choroidal vasculopathy with vitreous haemorrhage can rarely present as hyphema.

- Thorough work-up is warranted to identify the underlying cause.

- Management of hyphema is usually conservative; subsequent pars plana vitrectomy is needed for clearing of vitreous haemorrhage.

Contributors AK: concept and design. SKP, RD, KS: design, data collection and review.

Funding The authors have not declared a specific grant for this research from any funding agency in the public, commercial or not-for-profit sectors.

Competing interests None declared.

Patient consent Obtained.

Provenance and peer review Not commissioned; externally peer reviewed.

\section{REFERENCES}

1 Narayanan R, Mithal K, Jalali S, et al. Vitreous haemorrhage in massive hemorrhagic polypoidal choroidal vasculopathy: clinical characteristics and surgical outcomes: vitreous hemorrhage in PCV. Int J Retina Vitreous 2015;1:25.

2 Lin HC, Yang CH, Yang CM. Medscape. Visual outcomes of vitrectomy for polypoidal choroidal vasculopathy-related breakthrough vitreous haemorrhage. Eye 2014;28:797-807.

3 Kwon HJ, Sung Y, Song WK. Massive hemorrhagic complications after intravitreal injection of aflibercept in patients with presumed polypoidal choroidal vasculopathy. J Clin Exp Ophthalmol 2015;6:451-4.

Copyright 2018 BMJ Publishing Group. All rights reserved. For permission to reuse any of this content visit

https://www.bmj.com/company/products-services/rights-and-licensing/permissions/

BMJ Case Report Fellows may re-use this article for personal use and teaching without any further permission.

Become a Fellow of BMJ Case Reports today and you can:

- Submit as many cases as you like

- Enjoy fast sympathetic peer review and rapid publication of accepted articles

- Access all the published articles

- Re-use any of the published material for personal use and teaching without further permission

For information on Institutional Fellowships contact consortiasales@bmjgroup.com

Visit casereports.bmj.com for more articles like this and to become a Fellow 